\title{
Mechanisms of action and resistance for multiple myeloma novel drug treatments
}

\author{
Shinsuke Iida ${ }^{1}$
}

Received: 5 April 2016 / Revised: 2 June 2016 / Accepted: 2 June 2016 / Published online: 9 June 2016

(C) The Japanese Society of Hematology 2016

Multiple myeloma (MM) is an incurable plasma cell malignancy that develops as a result of multistep tumorigenic events [1]. The median overall survival of patients with newly diagnosed MM was approximately 2.5-3 years when melphalan and prednisone (MP) therapy was the standard of care. However, since the end of the 20th century, genetic and epigenetic alterations associated with the pathogenesis of MM and tumor cell biology of the bone marrow microenvironment have been gradually unraveled [2, 3]. In parallel, the discovery of thalidomide as an active agent for MM in 1999 ushered in a new era in the treatment of this intractable disease [4]. This was followed by the clinical application of bortezomib, a first-generation proteasome inhibitor (PI), based on the results of pre-clinical translational studies $[3,5]$. Novel thalidomide derivatives called immunomodulatory drugs (IMiDs), such as lenalidomide and pomalidomide, were also developed [6, 7]. Second-generation PIs, such as carfilzomib and ixazomib, have recently been approved by the FDA [8]. Currently, PIs and IMiDs, as well as corticosteroid and alkylating agents, are the key novel drugs for MM treatment which have resulted in the achievement of a median survival of approximately 5-6 years [9]. Moreover, additional agents with different mechanisms of action have been incorporated into the treatment of $\mathrm{MM}$, including the deacetylase inhibitor panobinostat and monoclonal antibodies, targeting SLAMF7, CD38, and PD-1 represented by elotuzumab, daratumumab/isatuximab, and pembrolizumab/nivolumab,

Shinsuke Iida

iida@med.nagoya-cu.ac.jp

1 Department of Hematology and Oncology, Nagoya City University Graduate School of Medical Sciences, 1 Kawasumi, Mizuho-cho, Mizuho-ku, Nagoya, Aichi 467-8601, Japan respectively $[10,11]$. Thus, the prognosis of patients with MM is expected to continue to improve.

Interestingly, however, the mechanisms of action of these new agents for MM have not been fully elucidated. These new agents are completely different from such drugs as tyrosine kinase inhibitors (TKIs), which are used in the treatment of chronic myeloid leukemia (CML), because the target molecule is not well specified [12]. When thalidomide was induced, the inhibitory effect on angiogenesis and TNF $\alpha$ production were considered attributable to its antiMM activity. When the PI bortezomib was being studied in clinical trials, it was believed that the inhibitory effect on NF- $\kappa$ B signaling, via the prevention of degradation of poly-ubiquitinated $\mathrm{I} \kappa \mathrm{B}$, was attributed to the robust killing activity on MM cells. Sparked by the great discovery that cereblon was the molecule responsible for binding to thalidomide and causing its teratogenicity, it is now known that IMiDs induce the apoptosis of MM cells through the down-regulation of IRF4, via the cereblon-dependent ubiquitination of IKZF1 (Ikaros) and IKZF3 (Aiolos) which are transcription factors that are known to play an essential role in lymphocyte differentiation [13, 14]. Poly-ubiquitinated IKZF proteins caused by the altered substrate specificity of cereblon are degraded in the proteasome, resulting in the decreased transcription of IRF4 [15, 16]. At least one of the main mechanisms of bortezomib responsible for inducing apoptosis in MM cells is now considered to be excessive ER stress triggered by proteasome inhibition [17, 18]. The deacetylase inhibitor panobinostat not only inhibits major classes of histone deacetylases (HDAC), but also inhibits HDAC6, which is involved in the aggresome pathway and acetylates various critical proteins, such as TP53, Hsp90, and HIF-1 $\alpha$ [19]. Thus, the critical mechanisms of action of these novel agents in MM cells in association with the 
bone marrow microenvironment have just begun to be understood.

The complete elucidation of the mechanisms of action of novel agents used in MM treatment is important for two reasons. First, the patients with MM eventually relapse after novel agent-containing therapies and they often show acquired resistance against these agents at the time of relapse. Even though this is a crucial clinical problem, the detailed mechanisms responsible for this type of resistance are unknown. To elucidate the molecular mechanisms of action for each novel agent is expected to lead to figuring out a treatment strategy to overcome this resistance. Second, the elucidation of the mechanisms of action for each agent can lead to the development of additional novel drugs and identify novel target molecules in MM cells and the bone marrow microenvironment. In other words, true molecular targeting drugs for MM, which are like the TKIs in CML, could be developed in the near future.

In this review series in Progress in Hematology, we have invited four authors who are actively involved in basic or translational research focused on this issue.

\section{Compliance with ethical standards}

Conflict of interest S.I. received an honorarium from Celgene K.K., Janssen Pharmaceutical K.K., Bristol-Myers Squibb, Takeda Pharmaceutical Co. Ltd., and Ono Pharmaceutical Co. Ltd. S.I. also received research funding from Kyowa Hakko Kirin Co. Ltd., Chugai Pharmaceutical Co. Ltd., Eli Lilly Japan K.K., Celgene K.K., Takeda Pharmaceutical Co. Ltd., Ono Pharmaceutical Co. Ltd., Janssen Pharmaceutical K.K., Novartis Pharma K.K., Teijin Pharma, Astellas Pharma Inc., Toyama Chemical Co. Ltd. Bayer Yakuhin Ltd., and Sanofi K.K.

\section{References}

1. Iida S, Ueda R. Multistep tumorigenesis of multiple myeloma: Its molecular delineation. Int J Hematol. 2003;77:207-12.

2. Chapman MA, Lawrence MS, Keats JJ, Cibulskis K, Sougnez $\mathrm{C}$, Schinzel AC, et al. Initial genome sequencing and analysis of multiple myeloma. Nature. 2011;471:467-72.

3. Hideshima T, Mitsiades C, Tonon G, Richardson PG, Anderson KC. Understanding multiple myeloma pathogenesis in the bone marrow to identify new therapeutic targets. Nat Rev Cancer. 2007;7:585-98.

4. Singhal S, Mehta J, Desikan R, Ayers D, Roberson P, Eddlemon $\mathrm{P}$, et al. Antitumor activity of thalidomide in refractory multiple myeloma. N Engl J Med. 1999;341:1565-71.
5. Richardson PG, Barlogie A, Berenson J, Singhal S, Jagannath S, Irwin D, et al. A phase 2 study of bortezomib in relapsed, refractory myeloma. N Engl J Med. 2003;348:2609-17.

6. Dimopoulos MA, Spencer A, Attal M, Prince HM, Harousseau JL, Dmoszynska A, et al. Lenalidomide plus dexamethasone for relapsed or refractory multiple myeloma. N Engl J Med. 2007;357:2123-32.

7. San-Miguel J, Weisel K, Moreau P, Lacy M, Song K, Delforge $\mathrm{M}$, et al. Pomalidomide plus low-dose dexamethasone versus high-dose dexamethasone alone for patients with relapsed and refractory multiple myeloma (MM-003): a randomized, openlabel, phase 3 trial. Lancet Oncol. 2013;14:1055-66.

8. Dimoloulos MA, Moreau P, Palumbo A, Joshua D, Pour L, Hajek $\mathrm{R}$, et al. Carfilzomib and dexamethasone versus bortezomib and dexamethasone for patients with relapsed or refractory multiple myeloma (ENDEAVOR): a randomized, phase 3, open-label, multicenter study. Lancet Oncol. 2016;17:27-38.

9. Kumar SK, Dispenzieri A, Lacy MQ, Gertz MA, Buadi FK, Pandey $\mathrm{S}$, et al. Continued improvement in survival in multiple myeloma: changes in early mortality and outcomes in older patients. Leukemia. 2014;28:1122-8.

10. San-Miguel JF, Hungria VT, Yoon SS, Beksac M, Dimoloulos MA, Elghandour A, et al. Panobinostat plus bortezomib and dexamethasone versus placebo plus bortezomib and dexamethasone in patients with relapsed or relapsed and refractory multiple myeloma: a multicenter, randomized, double-blind phase 3 trial. Lancet Oncol. 2014;15:1195-206.

11. Lonial S, Durie B, Palumbo A, San-Miguel J. Monoclonal antibodies in the treatment of multiple myeloma: current status and future perspectives. Leukemia. 2016;30:526-35.

12. Santos FP, Kantarjian H, Quintas-Cardama A, Cortes J. Evolution of therapies for chronic myelogenous leukemia. Cancer J. 2011;17:465-76.

13. Ito T, Ando H, Suzuki T, Ogura T, Hotta K, Imamura Y, et al. Identification of a primary target of thalidomide tetragenicity. Science. 2010;327:1345-50.

14. Yoshida T, Georgopoulos K. Ikaros fingers on lymphocyte differentiation. Int J Hematol. 2014;100:220-9.

15. Fisher ES, Böhm K, Lydeard JR, Yang H, Stadler MB, Cavadini $\mathrm{S}$, et al. Structure of the DDB1-CRBN E3 ubiquitin ligase in complex with thalidomide. Nature. 2014;512:49-53.

16. Chamberlain PP, Miller K, Carmel G, Pagarigan B, Chie-Leon B, Rychak E, et al. Structure of the human cereblon-DDB1-lenalidomide complex reveals basis for responsiveness to thalidomide analogs. Nat Struct Mol Biol. 2014;21:803-9.

17. Leung-Hagesteijn C, Erdmann N, Cheung G, Keats JJ, Stewart $\mathrm{AK}$, Reece DE, et al. Xbp1 s-negative tumor B cells and preplasmablasts mediate therapeutic proteasome resistance in multiple myeloma. Cancer Cell. 2013;24:289-304.

18. Ri M, Iida S, Nakashima T, Miyazaki H, Mori F, Ito A, et al. Bortezomib-resistant myeloma cell lines: a role for mutated PSMB5 in preventing the accumulation of unfolded proteins and fetal ER stress. Leukemia. 2010;24:1506-12.

19. Hideshima T, Bradner JE, Wong J, Chauhan D, Richardson P, Schreiber SL, et al. Small-molecule inhibition of proteasome and aggresome function induces synergic antitumor activity in multiple myeloma. Proc Natl Acad Sci USA. 2005;102:8567-72. 